\title{
Gold Coatings for Temperature Control in Space Exploration
}

\author{
Robert C. Langley \\ Engelhard Industries, Newark, New Jersey
}

Thin coatings of gold, applied by a variety of techniques, are used extensively for protection against the effects of radiant energy in space exploration. Further applications in space technology appear to be likely, based on the ease with which the intrinsic properties of gold can be utilised to meet a variety of thermal control problems, while the techniques developed may well be of value in the control of radiant energy in industrial applications on earth.

Certain applications of gold in space technology have been referred to in recent articles in the Gold Bulletin in connection with discussions of the uses of gold in brazing alloys and in electronics. This article will describe the applications of gold coatings for temperature control in space and will explain why gold has become the material of choice for a variety of temperature control uses.

Heat can be transferred, in the absence of a working fluid, only by conduction or radiation. The use of a working fluid involves piping, means of circulation and a radiator. In space technology, these are avoided wherever possible in order to minimise weight or system complexity, or because of the risk of failure of any system that has moving parts. For the control of radiant energy in space, the U.S. engineering team decided to use stable inorganic coatings. This approach is called "passive temperature control" and it is in these coatings that gold has unique utility.

The wavelength of peak radiation from any source being dependent on its temperature, objects at moderately high temperature (e.g. $800^{\circ} \mathrm{C}$ to $1000^{\circ} \mathrm{C}$ ) have peak radiation in the short wavelength infrared region (from one to 15 microns). Very hot objects, such as the sun, have peak radiation at
The Lunar Module of the Apollo 14 mission on the Fra Mauro landing site. Reflective foil made of gold on plastic protects the vehicle and its equipment from solar radiation in the airless conditions of space

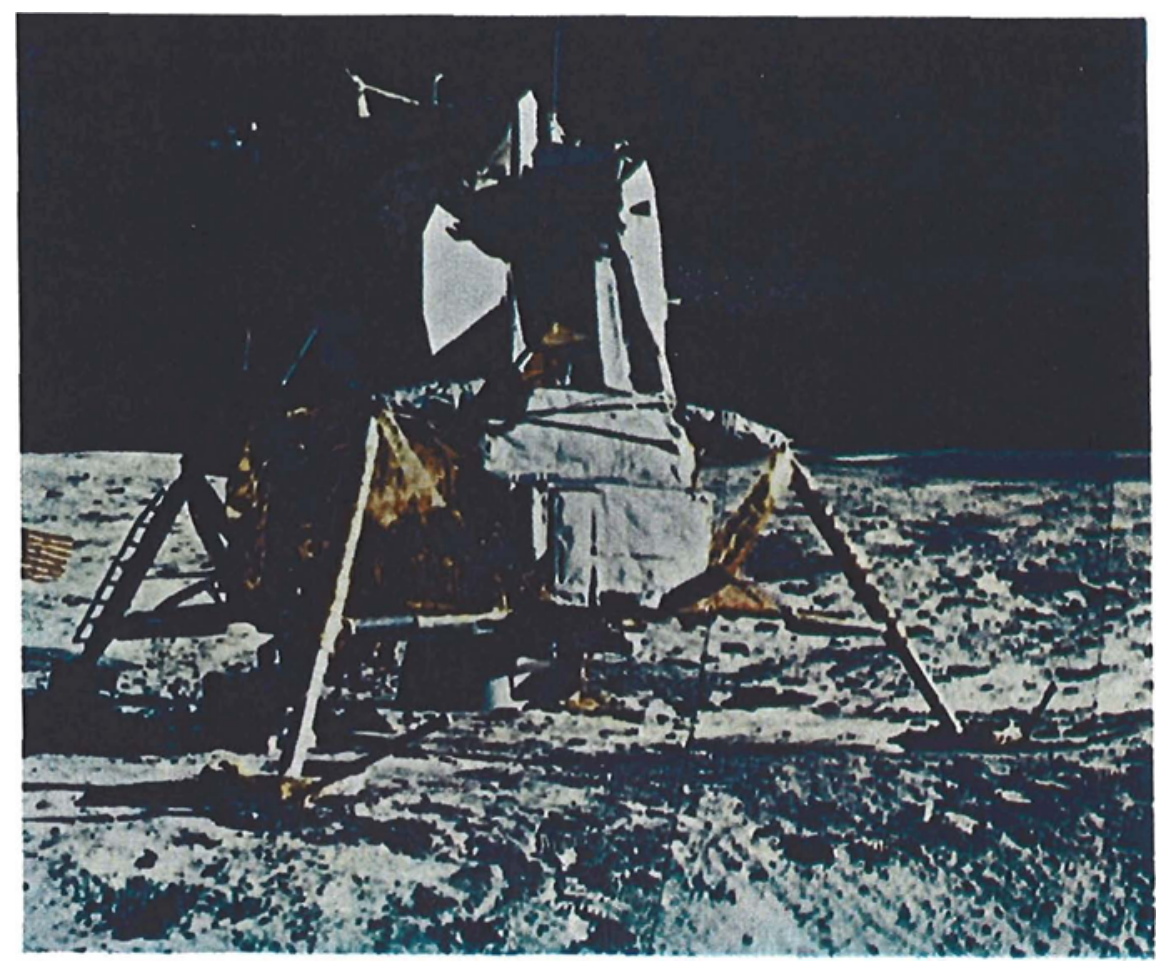




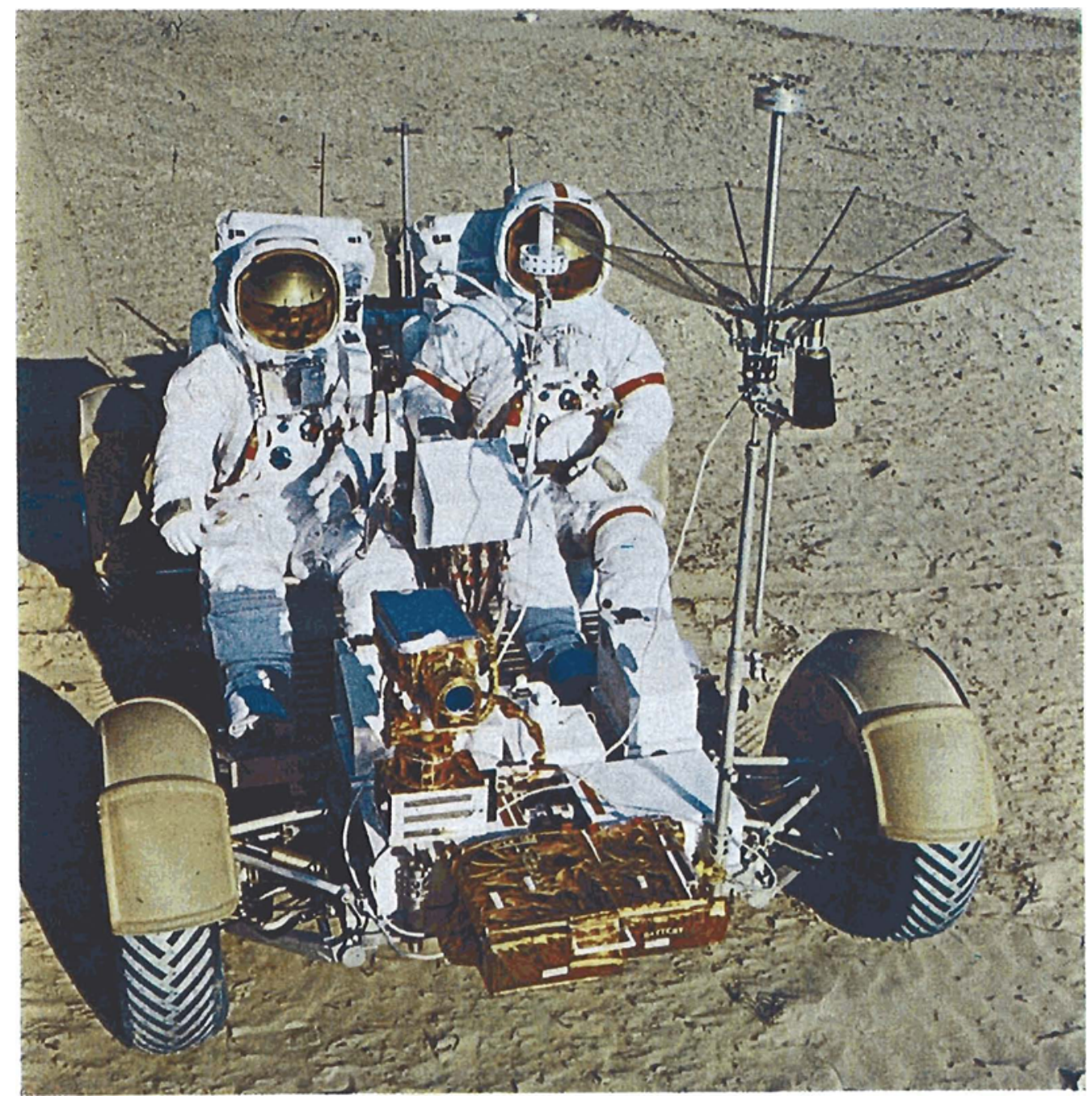

Astronauts Scott and Irwin in the Lunar Rover used in the Apollo 15 misgion. The plastic vigors of their helmets have a thin gold film, applied by thermal evaporation in vacuum to reduce the glare from sunlight while permitting good visibility. The television camera and the batteries are covered with a gold-on-plastic reflecting film to maintain them at an even temperature

shorter wavelengths. The wavelength of peak solar radiation is about 0.5 microns, in the visible band. The designer of temperature control coatings for use in space is thus concerned principally with radiation wavelengths from about 0.2 to 15 microns.

One way to control the temperature of a surface exposed to this radiation is to coat the surface with a reflector. At these wavelengths, the best reflector materials are aluminium, silver, copper and gold. The four metals are approximately equal in reflecting infra-red, while aluminium and silver are superior to the coloured metals in reflecting visible energy. Silver and copper cannot be used since these metals would form sulphides prior to reaching space and this would result in complete loss of reflective properties. Gold is preferred over aluminium for many reflector applications in space primarily because of the variety of methods by which it can be applied to many substrate materials. The availability of these methods of applying gold, all of which are fully developed, is in sharp contrast to the situation with aluminium coatings, which can be applied only by thermal evaporation or sputtering or by a rather difficult method such as pack cementation.

All these techniques for the application of thin gold coatings to a wide range of substrates have been the subject of a great deal of investigation over the past few years, in part of course in specific connection with the NASA space programme, and very sophisticated methods are now available. The vacuum deposition processes of thermal evaporation and cathodic sputtering - processes that were first used with gold - have become more fully understood, while major progress has been made in the development of plant and equipment (1). 


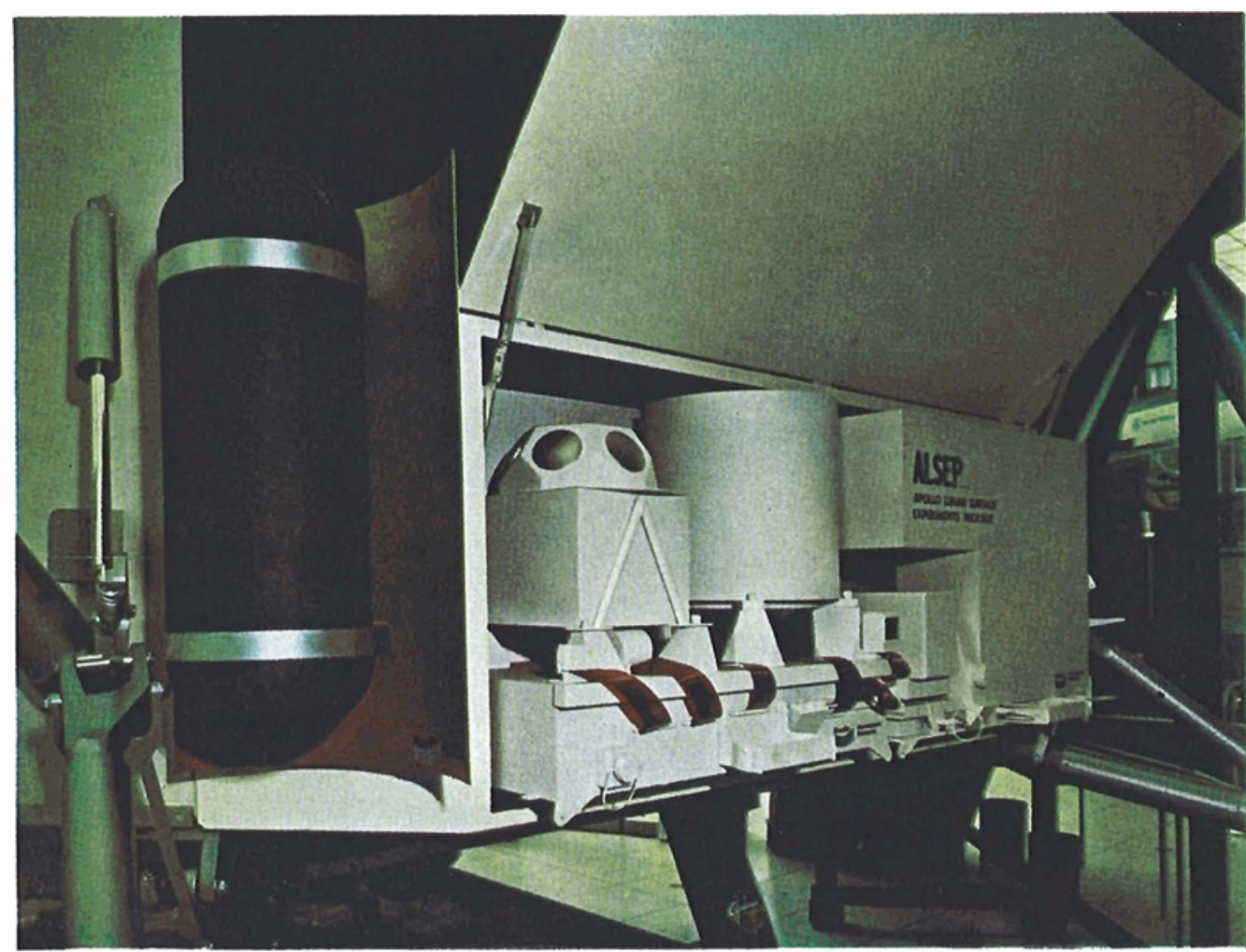

The power source of the Apollo Lunar Surface Experiments Package (ALSEP) is thermoelectric with the heat supplied from a radioactive fuel capsule on the left of the illustration. To reflect the heat away from the numerous instruments in the package - and also away from the spacecraft while it journeys to the moon - a curved titanium reflector, electroplated with gold, partially surrounds the capsule

Electroplating with gold is, of course, a very oldestablished process, but here again considerable research has had to be carried out to ensure complete reliability and to provide, for example, a suitable diffusion barrier between a titanium substrate and the final gold electrodeposit, when a reflector is subjected to high temperature conditions for long periods.

A further technique, the "liquid bright gold" method, relies upon the use of solutions of complex gold compounds in organic solvents. These can be produced with suitable viscosities for application by spraying or other means to a wide range of substrates. A carefully controlled heating operation will then decompose the organic materials leaving a firmly adherent film of gold. To meet the specialised needs of the space programme a great deal of work has been done on developing suitable formulations of these liquid golds.

A method for the deposition of gold films on both metallic and non-metallic surfaces by spraying with an aqueous solution of a gold salt complexed with an aliphatic amine has also been developed. The surface is simultaneously treated with a reducing solution containing hydrazine and an adherent film of metal is produced (2). The method is rapid and has the further advantages that large substrates can be coated and that no heating is required.

The use of gold as a reflective coating can be achieved at minimum cost by employing films just "thick" enough to develop full reflective properties so that transmission is zero. This thickness has been measured using thin gold films on glass and found to be about 1000 Angstroms ( 0.000004 inches). Thus about two grams of gold (costing about $\$ 2.50$ ) are needed to cover one square metre with a film $1000 \AA$ in thickness.

A gold reflector is used on the visors of the astronauts' space helmets. The same type of reflector has been used in military aviation for about a decade. In this application, the reflective film serves a dual purpose as it is in addition an optical coating to reduce glare from sunlight while admitting sufficient light for good vision. This partially transparent film is about $500 \AA(0.000002$ inches $)$ in thickness. It is applied by thermal evaporation in vacuum to the visor, made of acrylic plastic. This use can be seen in the illustration on page 63, which also shows gold-on-plastic reflectors on the television camera and on the batteries of the Lunar Rover.

A gold reflector has been used with the Apollo Lunar Surface Experiments Package (ALSEP). 
This is a power source for a number of instruments, designed to be landed on the moon by astronauts. The power unit is thermoelectric, with heat supplied by a radioactive source, and it will supply power for at least one year. Heat generated by the radioactive fuel must be reflected away from the instruments and the spacecraft en route to the moon. This was accomplished by placing a curved titanium reflector between the heat source and the instruments to reflect this energy into space. This reflector is shown in the illustration opposite, partially surrounding the radioactive fuel capsule. In this application the gold coating was applied by electroplating over a thin diffusion barrier layer. The refiector absorbs a fraction of the energy raising the temperature of the gold coating. The barrier layer is necessary to prevent interdiffusion between gold and titanium and consequent loss of the reflective property of pure gold.
In a different passive temperature control application, gold is used, not as a reflector, but to contain heat, utilising the fact that gold is a low emitter of radiant energy. The utility of gold in these applications is evident from the following equation for emission of radiant energy from any heated surface.

$W=\varepsilon \sigma\left(T^{4}-T_{0}^{4}\right)$

where $W$ is the radiant flux

$\varepsilon$ is the emissivity of the surface

$\sigma$ is the Stefan-Boltzman constant

$T$ is the absolute temperature of the surface $T_{0}$ is the absolute temperature of the surroundings

Emissivity is expressed on a scale where $1.0=100$ per cent, and the value for gold is very low, 0.05 . Most structural metals oxidise readily, resulting in oxide coatings of high emissivity. For example, oxidised Inconel has an emissivity of about 0.8 , high

The pressure vessels of the fuel cells used to supply power in the Apollo spacecraft must be maintained at constant temperature to keep the reactants - hydrogen and oxygen - at constant pressure. Loss of heat from these vessels is avoided by wrapping them with a flexible plastic film coated with a liquid gold preparation produced by Engelhard Minerals and Chemicals. This illustration shows the gold film insulation being secured around the pressure vesgels at Pratt and Whitney

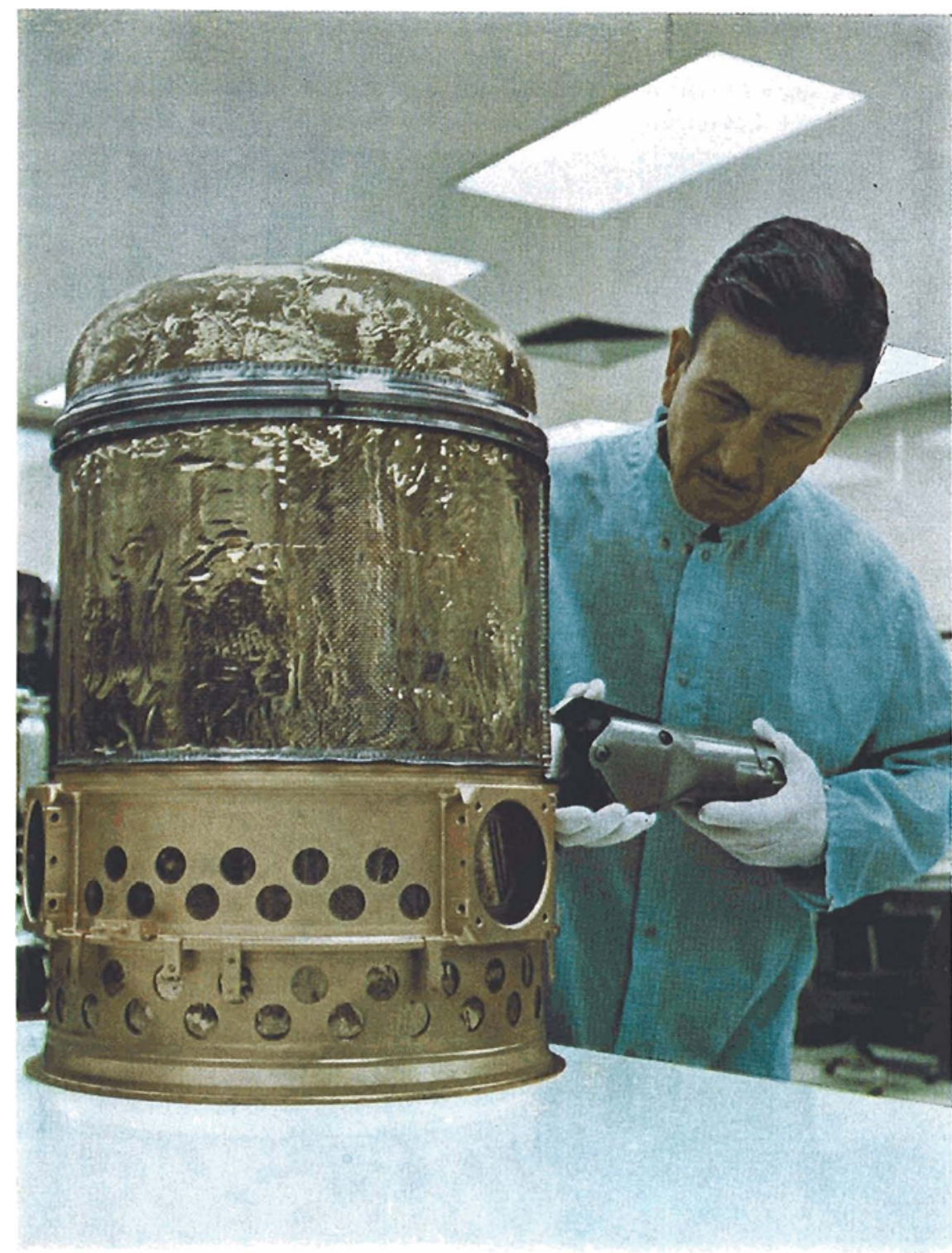


enough for use as a black body standard in emissivity measuring instruments. In comparing two surfaces, at the same temperature and radiating to the same environment, it can be seen that a gold coated surface will radiate only $0.05 / 0.8$ of the amount of energy radiated by an oxidised Inconel surface. This property of gold was used to maintain radioactive fuel capsules at design temperature in thermoelectric power sources soft-landed on the moon before the first manned landing. The substrate was a nickelchromium alloy and gold was applied by electroplating.

For the fuel cells used to supply electrical power in the Apollo spacecraft gold was also used as a low emissivity coating. The pressure vessels of the fuel cells must be maintained at constant temperature to keep the fuel cell reactants, hydrogen and oxygen, at constant pressure. Loss of heat by radiation from the pressure vessels is prevented by wrapping the vessels with a flexible plastic film, coated with a gold film about $1000 \AA$ in thickness. In this use, gold was applied to the plastic by the "liquid bright gold" method using a formulation developed by Engelhard Minerals and Chemicals. The formulation was a solution for application by spraying, containing gold $t$-dodecyl mercaptide, a compound which decomposes to metal at $150^{\circ} \mathrm{C}$. After spraying, the gold film was developed by infra-red heating.

In the early days of the American space programme, there was no certainty that fuel cell power sources could be developed with sufficient reliability, and alternate approaches to power sources for manned space flight were considered. In one approach a solar energy collector was considered, utilising a thin gold film having a unique combination of energy transfer properties. The goal was to develop a coating to absorb solar radiation and capable of operating for thousands of hours at 700 to $800^{\circ} \mathrm{C}$. The coating was to be heated to this temperature by solar energy concentrated by a suitably shaped reflector. The substrate for the coating was Inconel, which served as the hot junction of a thermoelectric power source.

As noted above, oxidised Inconel is a black body and would seem to be an excellent absorber of solar radiation. However, all black bodies are also excellent emitters of radiation. Oxidised Inconel absorbs solar wavelengths (the band from about 0.3 to 1.5 microns) and is heated. It then emits at longer wavelengths resulting in a relatively low equilibrium temperature.

Gold has very low emissivity and research was undertaken to develop a composite gold film that would have this desirable property, but which would also absorb wavelengths of peak solar radiation. This development was successful and resulted in a film containing 89.5 per cent gold by weight and carefully balanced amounts of oxides of bismuth, barium, chromium and silicon $(3,4)$. The composite film was very thin, about $2000 \AA$ in thickness; it was applied to Inconel by the "liquid bright gold" method. Rapid advances in fuel cell technology, however, rendered solar powered thermoelectric systems unattractive for manned space missions.

This article has described some of the intrinsic properties of gold which make it useful in temperature control coatings in space. Future applications seem possible based on the ease with which intrinsic properties can be varied to suit specialised thermal control needs. Above all, the wide variety of methods by which gold can be applied to almost any substrate material make this metal very useful in coatings for space, while the developments outlined in this article show promise of leading to industrial applications on earth.

\section{References}

1 Gold Bulletin, 1971, 4, (2), 30-32

2 U.S. Patent 3,515,571, Donald J. Levy, assigned to Lockheed Aircraft Corp.

3 U.S. Patent 3,176,678, Robert C. Langley, assigned to Engelhard Industries Inc.

4 U.S. Patent 3,176,679, Robert C. Langley, assigned to Engelhard Industries Inc.

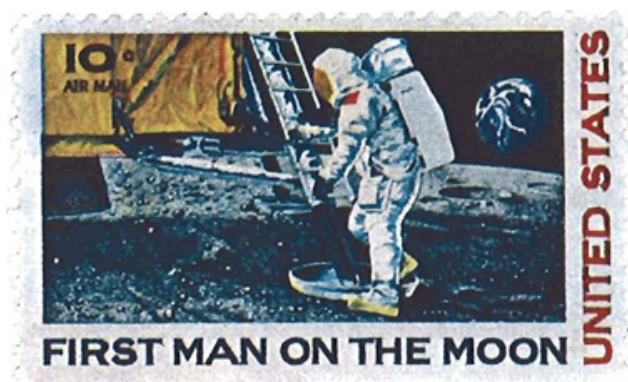

This ten-cent stamp issued by the United States Post Office commemorates the first Moon landing and clearly illustrates the reflective gold coating on the Apollo Lunar Module as well as the evaporated gold film on the visor of the suits worn by the astronauts to reduce glare while maintaining good visibility 